$\begin{array}{ll}\text { le portiQue } & \text { Le Portique } \\ \text { Revue de philosophie et de sciences humaines }\end{array}$

$11 \mid 2003$

Le Respect

\title{
Respect et vulnérabilité chez Levinas
}

\author{
Nicolas Antenat
}

\section{OpenEdition}

\section{Journals}

Édition électronique

URL : http://journals.openedition.org/leportique/558

DOI : 10.4000/leportique.558

ISSN : $1777-5280$

\section{Éditeur}

Association "Les Amis du Portique"

Édition imprimée

Date de publication : 1 janvier 2003

ISSN : 1283-8594

\section{Référence électronique}

Nicolas Antenat, «Respect et vulnérabilité chez Levinas », Le Portique [En ligne], 11 | 2003, mis en ligne le 15 décembre 2005, consulté le 25 mars 2021. URL : http://journals.openedition.org/leportique/558 ; DOI : https://doi.org/10.4000/leportique.558

Ce document a été généré automatiquement le 25 mars 2021

Tous droits réservés 


\title{
Respect et vulnérabilité chez Levinas
}

\author{
Nicolas Antenat
}

" Respect et vulnérabilité » peut apparaître comme un titre facile et aguichant, allant de soi en quelque sorte. Il est, en effet, aisé de réduire l'association de ces deux termes au registre du pathos, des sentiments compassionnels, misérabilistes. Cela reviendrait, finalement, comme il est coutume de le faire, à réduire la pensée de Levinas à une certaine forme de religiosité quelque peu laïcisée et rationalisée, tout entière tournée vers l'amour du prochain. L'enjeu, nous allons le voir, est bien plus fondamental.

Bien entendu, nous éprouvons du respect, entendu dans son sens le plus simple, c'est-àdire de la considération, pour " la veuve et l'orphelin », en regard de leur vulnérabilité, de leur disposition à être blessé, atteint. Nous en faisons, si nous échappons à la distraction générale, l'expérience quotidienne. Mais cette épreuve du respect, au-delà de la compassion et de la sympathie, nous fait ressentir comme une fêlure que nous avons généralement bien du mal à formaliser. Or, c'est par et à travers cette fêlure, cette percée, cette rupture que Levinas va chercher à comprendre et à définir la subjectivité dont le statut est assurément le thème central de sa réflexion polyvalente et ambiguë, pensée ambiguë pour penser l'ambiguïté elle-même. Cependant, la subjectivité, lorsqu'elle n'est pas divertie, n'est pas limpide d'elle-même, ne va pas sans problème, nous allons le voir. Vulnérabilité, elle ne s'érige qu'à la condition d'être altérée, dévastée par la rencontre de l'autre. Dans la même ligne que Scheler, Buber, Rosenzweig et d'autres, Levinas fait dépendre en droit la subjectivité de l'intersubjectivité.

3 C'est bien à ce travail et à cet itinéraire que nous invite l'œuvre de Levinas dans son ensemble. Pourquoi et comment passer d'une philosophie de la violence (et donc de l'irrespect) à une philosophie de la caresse (où l'autre est respecté dans son altérité). Il est trop facile de ne pas voir les enjeux réellement philosophiques qui se cachent derrière les descriptions apparemment naïves de la relation à l'autre homme faites par Levinas mais que Blanchot et beaucoup d'autres ont toujours pris très au sérieux: «Quand Levinas demanda si l'ontologie était fondamentale [...], il posait d'une certaine 
manière une question naïve, inattendue et inentendue, parce que rompant avec ce qui paraissait avoir renouvelé la philosophie et qu'il avait le premier contribué à comprendre et à transmettre, rompant ainsi avec lui-même. De même, et dans la suite de ce mouvement, quand il prononça le mot Autre et le rapport de moi à Autrui comme un rapport exorbitant, infini ou de transcendance, tel qu'on ne pouvait pas le ressaisir par une réflexion sur l'être et l'étant et tel que toute la philosophie occidentale s'en était détournée traditionnellement par le privilège accordé au Même, au Moi-Même ou plus abruptement à l'identité, la critique pouvait juger cette affirmation naïve, accumuler les objections, réfuter (comme il est dit de K. dans le Château : il a toujours été réfuté), c'est la critique qui était naïve, n'entendant pas ce qu'il y avait de décisif dans cette exigence, de difficile aussi, qui mettait mal à l'aise la raison, fût-elle pratique, sans cependant la renvoyer ${ }^{1}$.

4 Il y a bien, en effet, chez Levinas un appel et un rappel, un dégrisement, la volonté de rompre avec une philosophie du savoir sans pour autant négliger l'exigence jamais assez satisfaite de lucidité et de rationalité. Il y a bien dans cette pensée une contestation et une rupture de l'idée traditionnelle d'après laquelle le lieu naturel et l'origine même de la philosophie se trouverait «dans le psychisme humain entendu comme savoir allant jusqu'à la conscience de soi». C'est à une rupture avec une conception réductionniste de l'intelligibilité qui consisterait en l'intégration de l'Autre au Même, c'est-à-dire à la totalité, que nous invite le philosophe.

5 Certes, ce parcours risque, à bien des égards, de nous mener hors du champ de la philosophie dans son acception rigoureuse ${ }^{2}$, celle ouverte par les Grecs et qui trouve son apogée dans la phénoménologie husserlienne que Levinas qualifie de philosophie du Même. Le Même, dans le langage de Levinas, renvoie, pour aller vite, à la totalité. C'est le savoir théorique, l'objectivité, l'impérialisme de l'assimilation réductrice et violente: l'ontologie, la guerre, les philosophies du système (celle de Hegel en particulier). La répétition du Même, la totalité ou l'exigence de totalité forme les idéaux qui gouvernent la pensée occidentale: intégrer, unifier, totaliser, globaliser, etc., comme si l'esprit ne pouvait s'apaiser et trouver satisfaction qu'à travers des synthèses, l'unité et l'identité. À cette totalité prégnante et collante, Levinas oppose l'infini, l'autre dans son irréductible altérité, l'expérience d'une transcendance, ici et maintenant, sur le plan même de l'immanence, qui ne se réduit jamais à une expérience mystique, à une extase. « La transcendance dans l'immanence, Levinas est le premier à s'interroger sur cette étrange structure - la sensibilité, la subjectivité - et à ne pas se laisser satisfaire par le heurt de telles contrariétés " ${ }^{3}$. Mais, il est important de s'en souvenir, c'est par la philosophie, par son langage ou plutôt par les abords mal contrôlés de son langage et de sa méthode que Levinas affleure les limites de la philosophie pour tenter une phénoménologie de l'imphénoménologisable. «La philosophie, fût-elle de rupture, nous sollicite philosophiquement ${ }^{4}$.

6 Ne l'oublions pas, Levinas est en fait un grand pourfendeur des mythes contemporains : l'esthétisme, les conceptions fusionnelles de l'amour et de la société, les "valeurs " comme refuge, les emportements ontologico-poétiques de la scène philosophique telle qu'elle est ordinairement: jonchée de penseurs ivre-morts de métaphysique, de «logos », d'être et de substance, de conscience et de liberté. Levinas est un solitaire venant des petits matins dégrisés, frappe à la porte de la taverne et crie qu'il est temps 
de fermer, ou de se réveiller, de prendre le pharmakon de la vigilance. «Rien n'est plus théâtre, le drame n'est plus jeu. Tout est grave " .

7 Son écriture, toujours amène, est, elle aussi, faussement séduisante. Derrière les jolis mots (le vulnérable, le tendre, la caresse, etc.), sourd à toute force un dégrisement radical, le travail d'un homme qui a exploré les différents domaines de la pensée. Avec Levinas, si on le lit, c'est un appel à la vigilance, au réveil et à la veille, « ... appel de la raison à l'éveil d'une autre raison... " ${ }^{6}$. Son œuvre ébranle et inquiète les demeures et les lieux habituels de la philosophie. Sa pensée « étrangère à tout mandarinat » met au centre les confins de la philosophie, se préoccupant de ce que cette dernière omet de dire, ce qu'elle a, depuis l'origine, en aversion. Pas question de se laisser séduire ou attendrir, même par la misère, la pauvreté extrême ou quotidienne, la veuve, l'orphelin. Certes, l'autre est vulnérable, et je peux en ce sens et pour cette raison, lui devoir du respect, nous l'avons dit. Or, ce que cherche à montrer Levinas c'est que ce respect n'émane pas d'une conscience souveraine ou de l'obéissance à une quelconque loi gommant toute aspérité. C'est l'Autre qui m'intime du respect. "Je suis l'otage de l'autre", phrase clé de l'éthique levinassienne, n'est pas une phrase d'éthique quotidienne; elle témoigne d'un excès qui arrache à la rationalité universelle. La rencontre d'autrui dans sa corporéité et ce qui la traverse, le visage, est la condition singulière qui ouvre sur l'excès, alors que l'universalité interdit cet excès, elle occupe toute la place. Et, occuper toute la place est dans la pensée du philosophe, une des formes fondamentales de l'irrespect.

Levinas reste fidèle à une expérience fondamentale, celle qui nous saisit lorsque nous ressentons le dénuement et l'extrême vulnérabilité d'un visage, vulnérabilité qui nous prend en otage dans le sens où, malgré nous, nous nous sentons responsables (sentir, éprouver cette responsabilité et l'assumer est une des formes primordiale de respect chez Levinas). Et il est vrai que chez lui, pour qui l'éthique est philosophie première, toute sa conception du sujet, de la subjectivité dépend de cette expérience. C'est l'éthique et non l'ontologie qui est première, c'est la relation intersubjective qui est première. Nous l'aurons compris, l'enjeu consiste à défaire la relation sujet-objet et le primat de la conscience, de l'ego et de la présence, afin de pouvoir prêter l'oreille au "dire » d'une sensibilité primaire et inépuisable, sensibilité sans retour à la maitrise d'un « je pense, donc tu n'es pas».

La rencontre du vulnérable, de l'autre dans son plus grand dénuement, est, chez Levinas, à l'image de la rencontre de l'idée d'infini pour Descartes. Que l'on donne pour l'un et l'autre comme origine de cette idée Dieu ou tout autre chose le problème n'est pas là et à vrai dire ne nous intéresse pas. Levinas se désintéresse de la Chose infinie. S'il revendique l'héritage de Descartes ce n'est pas parce que celui-ci thématise une substance infinie, incompréhensible - Dieu - mais avant tout parce qu'il décrit l'infinité comme enveloppant un débordement du Moi («ce en quoi je ne rencontre point de limites »). Et Levinas lit cet infini en moi avant tout comme un éclatement du contenant par le contenu. «Ce ne sont pas les preuves de l'existence de Dieu qui nous importent, mais la rupture de la conscience $»^{7}$.

10 L'expérience décisive, le trait décisif de cette expérience, c'est le débordement ou l'éclatement (de l'idée en tant que réalité formelle pour reprendre le langage cartésien). Ce débordement est ce qui désigne la structure même de la transcendance levinassienne. Or cette transcendance ne peut se définir que sur fond d'immanence, elle désigne précisément un débordement, une percée de l'immanence. La conscience est 
«désarçonnée, effondrée, rompue », «L'idée de l'infini signifie l'effondrement de la bonne conscience du Même " ${ }^{8}$. Ainsi, l'opposition entre l'idée du fini et l'idée d'infini est une opposition non pas entre deux idées de choses opposées mais entre ce qui relève du Même et ce qui précisément le déborde. Ce qui est visé donc ici c'est "l'inadéquation par excellence». Le respect et la vulnérabilité se jouent dans l'inadéquation entre moi et autrui.

11 Ce débordement, cet excès de l'autre sur la pensée qui le vise, peut être envisagé selon deux perspectives: objective et subjective. Objectivement, ce débordement désigne précisément le Visage dont «l'apparaître » consiste justement en un profond débordement du monde et du registre du Même comme tels. Nous y reviendrons. Mais ce débordement concerne en propre la subjectivité dont Levinas, nous insistons, renouvelle toute la conception. Cette conception conduit à souligner que le Moi est profondément débordé, excédé et que ce profond débordement du Moi désigne précisément la subjectivité, le Soi. La subjectivité se définit comme capacité d'accueil et accueil effectif de l'altérité : ce n'est plus « je est un autre » mais « le sujet est un hôte ». Contre une conception bien classique, la morale n'est pas un contrôle que la raison exerce sur la sensibilité, c'est un événement de la sensibilité. La morale n'est pas de l'ordre d'un devoir-être, c'est un fait, un traumatisme (celui que produit la rencontre du visage d'autrui).

12 Le visage, la rencontre du visage est ce qui me conduit hors de mon initiative et de mon pouvoir. Si celui-ci s'exprime dans le sensible, sa présence ne peut se résumer à cette présence sensible, adéquate au Moi. Cette présence consiste en une destruction incessante de celle-ci : «Le visage d'autrui détruit à tout moment, et déborde l'image plastique qu'il me laisse, l'idée à ma mesure - l'idée adéquate " ${ }^{2}$. Le visage a ceci de paradoxal que l'exigence éthique qui s'y exprime ou qui l'exprime se manifeste à travers la forme plastique de son apparence sensible. Pourtant il ne se laisse pas réduire à un apparoir, il déchire en permanence la forme plastique dans l'altérité parlante qui le constitue. Le visage donne le paradoxe d'une apparence sensible qui semble donner prise à l'appropriation et en même temps qui lui échappe radicalement.

Cette transcendance du visage qui perce le monde sensible ne conduit nulle part (être, monde, arrière-monde), car il relève précisément d'un transcender, d'une percée du lieu, du monde ou de l'être comme tel. La transcendance c'est l'inadéquation par excellence. Le visage (non-mondain) interrompt la conscience et ses pouvoirs et comme la présence du visage est inséparable d'un déploiement temporel, cette percée, cette interruption est incessante. Si le visage se donne d'abord "à la mesure de " (adéquatement à) la conscience puis perce son registre, il ne peut que la bouleverser. S'il n'y avait la rencontre - primordiale - du visage d'autrui, je pourrais vivre tranquille, dans la certitude de mon être et de mon pouvoir-savoir sur les choses; je serais puissant dans le règne de la phénoménalité. Mais le visage d'autrui vient rompre cet ordre ; soudain, j'ai face à moi non plus un objet que je peux connaître ou posséder mais l'irréductible, l'impressionnante présence du visage d'autrui qui se refuse à moi, et à toute interprétation. Bref la révélation du visage en tant que percée du registre du Même comme tel ne peut qu'instaurer une subjectivité comme conscience bouleversée et non pleine d'elle-même. Si la percée du visage est incessante, le bouleversement de la conscience le sera également et la subjectivité désignera précisément un tel bouleversement incessant. Parce qu'elle s'institue en face du visage, en tant qu'il se 
donne d'abord dans le registre du savoir, de la représentation, du Même, puis s'en arrache et en sort, les perce, la subjectivité d'abord absorbée par l'être, en sort.

L'analyse donnée dans Entre nous, précise bien que seul l'apparaître du visage d'autrui (du prochain) est inadéquat et implique en conséquence, une défection ou un renversement de la subjectivité comme conscience «le fait pour l'être de se vider de son être ", vulnérabilité. Cette défection ou défaite de l'identité du moi - ce qui peut à la rigueur se dire événement du soi-même -, précède tout événement subi ou mené par un sujet. Mais cela se situe en deçà, en deçà que Levinas exprime précisément dans le terme d'an-archie. Identité défaite jusqu'au bout.

Ainsi, si Levinas décrit l'obsession par autrui dans le visage, il s'attache ensuite à expliciter la signification pour la subjectivité de cette obsession. Dans la conscience de soi, jamais le tissu du même n'est rompu. Le retournement du Moi en Soi circonscrit au contraire « un traumatisme coupant le fil de la conscience ».

Ainsi la subjectivité réalise ces exigences impossibles : le fait de contenir plus qu'il n'est possible. «Contenir plus que sa capacité, c'est à tout moment faire éclater les cadres d'un contenu de pensée ${ }^{10}$. De cette manière, on peut dire de manière abrupte que la subjectivité peut être l'événement même de la transcendance parce que son ipséité ne se fige pas en identité ontologique. Chez Levinas la subjectivité du sujet se noue dans l'immédiateté du sensible (contre la prétention de l'idéalisme pour lequel la subjectivité est essentiellement pensée et concept). «L'immédiateté du sensible qui ne se réduit pas au rôle gnoséologique assumé par la sensation, est exposition à la blessure et à la jouissance, ce qui permet d'atteindre la subjectivité du sujet se complaisant en soi et se posant pour soi " ${ }^{11}$. La subjectivité est vulnérabilité du sensible, "adversité ramassée dans la corporéité susceptible de douleur dite physique, exposée à l'outrage et à la blessure, à la maladie et à la vieillesse, mais adversité dès la fatigue des premiers efforts du corps ${ }^{12}$.

17 La subjectivité est vulnérabilité, c'est-à-dire arrachement à la jouissance, dévouement à l'autre. L'immédiateté du sensible est l'exposition à autrui, la proximité du visage. La subjectivité est, malgré soi, pour l'autre. Dans le tréfonds de son intériorité, dans l'isolement de la jouissance, pointe déjà la signification éthique: l'immédiat de la sensibilité est sa vulnérabilité, son exposition à l'affection pour l'autre. La transcendance ne s'ajoute pas à la subjectivité, mais elle ne se réduit pas non plus à une illusion subjective. «Le pour-l'autre, ainsi, à la fois, contrarie le sujet et l'affect dans son intimité » .

Il y a donc déjà au sein du Même, de l'Autre. L'autre déjà obsède toujours, il est obsédant. Proximité et obsession sont ainsi des emphases ou des hyperboles de la présence de l'Autre dans le sujet.

Levinas n'abandonne pas le privilège de la présence, mais seulement celui de la présence à soi d'une conscience, d'une présence associée au Même et à son impérialisme, «La possession de soi dans un présent ». Le toujours-déjà-là d'autrui est encore une présence mais insaisissable par la conscience qui arrive toujours après coup, toujours en « retard ». La présence de l'autre est si immédiate qu'elle constitue la sensibilité, définie comme "exposition à l'autre ", si constante, si pressante, qu'elle est "obsession ", si proche qu'elle ne peut se montrer, qu'elle est la proximité même. La proximité, c'est la présence de l'autre au cœur du sujet en deçà de sa manifestation. « Le prochain n'est pas phénomène et sa présence ne se résout pas en présentation ». On 
peut dire que la proximité brûle l'étape de la conscience. «L'obsession n'est ni une modification ni une exaspération pathologique de la conscience, mais la proximité même des êtres. La conscience sous toutes ses formes [...] a déjà perdu cette présence proche ${ }^{13}$. On le voit, Levinas refuse la connotation pathologique de l'obsession, il l'entend bien plutôt comme l'ouverture la plus passive à l'autre même s'il demeure, dans ce traumatisme originaire, souffrance et fragilité.

Le bouleversement est grand. La subjectivité prend, dès lors, les caractères de l'extériorité. «Je suis d'emblée le serviteur du prochain, déjà en retard et coupable de retard, je suis comme ordonné du dehors - traumatiquement commandé sans intérioriser par la représentation et le concept l'autorité qui me commande. Cette persécution traumatisante est assumée comme l'essence de la subjectivité, toujours accusée et coupable et punie avant d'avoir rien fait " ${ }^{14}$. Ainsi, la persécution ne vient pas s'ajouter à la subjectivité mais fait partie du soi le plus intime, à la fois, paradoxalement, de l'essence du sujet et de l'essence de l'autre. Dans cette obsession incessante, pré-phénoménale, qui se loge au cœur de la subjectivité vulnérable, se trouve la responsabilité la plus haute. Le respect de l'autre ne relève pas d'une libre décision, il est toujours déjà là. «Le visage du prochain me signifie une responsabilité irrécusable, précédent tout consentement libre, tout pacte, tout contrat " ${ }^{15}$. Il n'y a pas ici de « fais comme si » ou de « en tant que ».

21 Être responsable (répondre de) pour l'autre par l'autre ce serait seulement être originairement exposé à la proximité du visage de l'autre. La responsabilité devient une forme originaire de la sensibilité, parce que toute sensibilité serait non pas d'abord marquée par le contact avec les choses, dans l'espace et le temps, mais d'abord par «l'exposition à l'autre». Secrètement, l'autre habiterait mon expérience sensible la plus élémentaire, celle même du manger et du boire, la jouissance comme la douleur.

Dans son travail, Levinas disjoint le concept de responsabilité de celui de liberté et d'intelligibilité. Il rattache la responsabilité à la passivité la plus radicale, à la sensibilité elle-même audacieusement ramenée à l'affection pour l'autre. Le respect dont la responsabilité pour l'autre est le fondement, n'est pas un exercice de la raison. Je ne suis pas libre de respecter autrui. La responsabilité, sans laquelle aucun respect n'est possible, s'éprouve antérieurement à tout choix. Ce que je "dois » faire pour l'autre m'est dicté par sa seule présence incontournable, par sa seule proximité et non pas un horizon d'altérité universelle. La responsabilité, et par conséquent l'éthique Levinassienne, n'est pas paradoxalement une relation ou une communication, mais un mouvement irrelatif, absolu, interne au sujet: le passage du Moi au Soi, du volontaire au non-volontaire.

Cette responsabilité est asymétrique et inaliénable, personne ne saurait me remplacer ${ }^{16}$. Pour le dire vite, elle est insupportable et pour ainsi dire inassumable. À ce propos, Levinas cite souvent la phrase de Dostoïevski dans les Frères Karamazov: "Chacun de nous est coupable devant tous pour tous et pour tout, et moi plus que les autres ». On a souvent glosé sur le caractère excessif de cette conception de la responsabilité. Mais Levinas l'assume car l'éthique est une exigence infinie. Exigence suprême le visage d'Autrui met en question l'heureuse spontanéité du moi, cette joyeuse force qui va comme il le dit dans Difficile liberté.

24 Cette vulnérabilité extrême, cette responsabilité totale expliquent les différentes façons de renoncer ou de louper l'expérience de l'altérité. Levinas les recense et explique à 
chaque fois, à travers ses textes, les raisons du ratage. Pour des raisons d'économie nous n'en retiendrons ici que deux : la connaissance qui procède par réduction de la différence et la relation amoureuse et voluptueuse (forme exemplaire de la rencontre de l'autre), toujours elle aussi menacée.

Soyons direct : connaître c'est toujours prétendre reconnaître pour s'y reconnaître. Le moi ne sort pas de lui-même, voyage de soi à soi. Levinas retrouve ici les analyses de Nietzsche et de Bergson: l'instinct de connaissance procède de l'instinct d'appropriation et de conquête.

Dans ce sens la philosophie de Levinas se présente avant tout, en toute révérence, comme contestation des conséquences de la phénoménologie de Husserl considérée comme une philosophie idéaliste qui pousse à l'extrême le primat de l'ego transcendantal. Rupture avec la conceptualité husserlienne (et ce qu'elle implique), la pensée de Levinas, procède à une singulière interruption, un suspens ou une époché de la phénoménologie elle-même.

De Husserl, que Levinas sera le premier à traduire en français et à introduire en France, il retiendra outre une admiration certaine, l'essentiel de la méthode phénoménologique, ce que l'on appellera la phénoménologie descriptive. Mais très vite l'opposition thématique avec Husserl est sévère. En fait l'auteur de Totalité et infini se lève contre l'auteur des Méditations cartésiennes pour décrire comment le moi est déterminé à partir de l'autre.

Si on prend le principe des principes tel qu'il est exprimé au $\S 24$ de Ideen I on lit: " toute intuition donatrice originaire est une source de droit de la connaissance; tout ce qui s'offre à nous dans l'intuition doit simplement être reçu pour ce qu'il se donne, mais sans non plus outrepasser les limites dans lesquels il se donne alors ». Précisément selon ce "principe des principes» (comme le souligne Marion dans Réduction et donation), «pour qu'une chose soit validée, légitimée, revendiquée, il faut et il suffit qu'elle se donne comme évidence au regard intuitif ». Au paragraphe 15 des Méditations cartésiennes Husserl écrit: «cette attitude nouvelle (la réduction phénoménologique) me fait voir que l'ensemble du monde et tout ce qui est en général n'est pour moi que quelque chose qui «vaut» pour moi, c'est-à-dire n'existe pour moi que comme cogitatum de mes cogitationes variables et liées entre elles dans cette variation même ». Ce «principe des principes» témoigne de ce qu'en phénoménologie les choses sont distinguées ou spécifiées non plus mondainement, à partir des attributs ou critères mondains qui les caractérisent, mais selon leur donation subjective: le phénoménologue analyse non pas ce qui apparaît - les choses en elles-mêmes - mais l'apparaître subjectif, la façon dont elles se donnent pour un sujet. L'intentionnalité est donation des choses elles-mêmes. Comme le rappelle Husserl dans L'Idée de la phénoménologie, résumé des cinq leçons (p.111), selon le cartésianisme «la connaissance est une prestation intérieure à l'âme, et c'est purement à l'intérieur de l'âme qu'elle produit des formations de connaissance ». Mais pour Husserl, penser une chose ne revient pas à en avoir, dans l'esprit, une image, une copie (mentale). Quand on pense une chose on la vise elle-même, en personne, comme chose réelle, comme étant effectif; le "principe des principes » le signifie en stipulant que l'intuition doit donner la «réalité corporelle» des choses. Telle est l'intentionnalité de la conscience : elle donne non pas un représentant de la chose intérieur à l'esprit mais les choses ellesmêmes, dans leur réalité corporelle. De plus si Husserl légitime de nombreux modes de donation, tous sont intentionnels. Telles est l'exclusivité husserlienne de 
l'intentionnalité : conformément au "principe des principes", ce que les différents modes de donation donnent est légitimé si et seulement s'ils s'accomplissent selon une évidence intuitive, c'est-à-dire selon le remplissement d'une visée intentionnelle. Dit autrement, la phénoménologie ne valide que les contenus se donnant - non pas seulement à une visée intentionnelle (vide de sens) - mais aussi par un regard intuitif en tant que remplissant la visée intentionnelle les visant. Une phrase de Husserl est à ce sujet significative : "Vouloir saisir l'univers de l'être vrai comme quelque chose qui se trouve en dehors de l'univers de la conscience... est absurde. Ils appartiennent essentiellement l'un à l'autre " ${ }^{17}$.

Si Husserl pose dans sa philosophie une certaine forme d'inadéquation et que Levinas n'a pas manqué de souligner (pour aller vite on peut dire que l'ego husserlien est intentionnel, immanent et non-spatial, et les choses naturelles sont nonintentionnelles, transcendantes et spatiales), du point de vue de Levinas le «principe des principes" conduit à une adéquation (assimilation) au donné qui "s'offre originairement à nous dans l'intuition ». Cette adéquation est celle classique du sujet et de l'objet: l'objet se donne intuitivement en comblant «la mesure de la visée, l'intentionnalité vide la remplissant». Cette adéquation témoigne pour Levinas du caractère de l'immanence (le Même) : «La transcendance de l'objet, en tant qu'elle est mesure de l'immanence du vécu (intuitif), revient pour ainsi dire à celle-ci. Cette façon, pour le réel, de se tenir dans une transcendance intentionnelle «à l'échelle » du vécu, et pour la pensée, de penser à sa mesure et ainsi de jouir, signifie immanence ${ }^{18}$. En ce sens, la transcendance de la chose relève du Même. Le Même désigne le Moi en tant qu'il s'approprie ou saisit ; un terme relève du Même, il revient au Même, s'il peut être saisit par le Moi. Pour Levinas cette saisie peut être théorique : la pensée pense à sa mesure, le savoir sait les choses qui, en tant que sues, perdent leur altérité et reviennent à l'immanence et au Même. D'où ces mots de Levinas: "L'autre se fait le propre du moi dans le savoir assurant la merveille de l'immanence", "réduire une réalité à son contenu de pensée c'est la réduire au Même ${ }^{19}$. S'expliquent dès lors les analyses de Levinas concernant l'intentionnalité ou la présence « en chair et en os » (la présence corporelle) exigée par le principe des principes husserliens : l'être est en donation et la donation est à entendre, nous dit Levinas, dans l'acception littérale de ce mot. Elle s'achève dans la main qui prend. C'est dans la prise en main que la chose ellemême s'égale à ce que l'intuition de la pensée voulait et visait.

Pour Levinas, c'est de l'exclusivité de l'intentionnalité que découle le manquement par Husserl de la véritable transcendance, c'est-à-dire de l'inadéquation par excellence. En somme, pour résumer son idée, avec Husserl la transcendance reçoit la signification de l'immanence. Cette adéquation, cette prise, signifie que le sujet peut s'approprier le monde, théoriquement et pratiquement et que ce dernier en conséquence relève de l'immanence et du Même. "L'immanence, c'est le monde, notre monde, le monde qui nous est donné et où l'autre n'est plus que le Même » ${ }^{20}$.

31 Levinas reconnaît à la phénoménologie husserlienne la profondeur de son analyse de la subjectivité, il en souligne l'originalité. Dans la mesure où elle dévoile, par-delà le sujet naturel, toujours adéquat à l'objet, un sujet inadéquat ou débordé, la réduction phénoménologique de Husserl tend pour lui, à des-absorber pour ainsi dire le sujet de l'être, à instaurer un sujet dépourvu de mondanité (l'ego transcendantal). Mais, très rapidement, il souligne que le primat de l'adéquation est maintenu dans cette sphère subjective transcendantale dévoilée par Husserl (au terme de la réduction). Ainsi, la 
plupart des analyses levinassiennes de la phénoménologie husserlienne déploient un même mouvement consistant, dans un premier temps, à reconnaître l'originalité de la pensée de Husserl de la subjectivité et dans un second temps à regretter qu'elle maintienne dans la sphère de la subjectivité transcendantale ce que la réduction semblait d'abord remettre en cause : le primat du savoir ou de l'adéquation, c'est-à-dire l'absorption dans le Même.

Ce primat correspond, selon Levinas, à l'esprit de la phénoménologie husserlienne qui est essentiellement animé par des préoccupations gnoséologiques. La réduction phénoménologique n'est-elle pas subordonnée comme l'écrit Levinas à «la recherche de la certitude ou de l'évidence adéquate " ${ }^{21}$.

D'après Husserl, autrui se phénoménalise selon ce qu'il appelle l'aprésentation " qui nous donne ce qui, en autrui, nous est accessible en original » ou qui légitime "une accessibilité indirecte mais véritable de ce qui est inaccessible directement en luimême ». En somme, s'il ne se donne pas en original à l'intuition, autrui, en demeurant une «modification intentionnelle de mon moi », ne remet pas en cause l'exclusivité de l'intentionnalité ou du savoir; en termes Levinassiens, "il ne désarçonne ou ne traumatise pas la conscience intentionnelle ou le savoir». Dans Notes sur le sens, en évoquant Husserl et cette $5^{\mathrm{e}}$ Méditation, Levinas écrit : « Mais à cette conception de la relation à autrui, nous ne lui reprochons pas seulement de s'obstiner à penser cette relation comme savoir indirect - incomparable certes à la perception où le connu se livre en « original » - mais de l'entendre encore précisément comme savoir ».

Nous avons vu que Levinas reprochait à la démarche husserlienne d'être essentiellement gnoséologique, donc de maintenir le privilège de l'adéquation et de l'immanence, et que c'est sur ce point que se séparent Husserl et Levinas: une comparaison du rapport à autrui met cette séparation singulièrement en évidence : alors que le visage d'autrui désigne précisément, comme nous le verrons, le seul point de percée de l'immanence, du registre du Même et de la connaissance, la $5^{\mathrm{e}}$ Méditation de Husserl présente, elle, autrui comme s'intégrant à un processus de connaissance, comme « moyen » de connaissance du monde objectif.

Sont ainsi suggérées, à travers ces analyses, les difficultés pour penser et pour décrire le Visage d'autrui, qui ne doit en aucune façon être considéré comme objet de savoir et de connaissance. "Il n'est pas sous catégories » ${ }^{22}$. Il ne peut même pas être catégorisé, thématisé ou connu comme percée, précisément parce qu'il désigne une percée de tout thème, de toutes catégories ou connaissances. La phénoménologie Levinassienne va trancher sur la phénoménologie husserlienne en ce qu'elle ne s'épuise pas, comme celle-ci, à thématiser le mode de donation des choses mais va s'attacher à décrire un mode spécifique de donation soigneusement distinct de celui des choses comme telles et « donnant » en conséquence, ce qui n'est plus une chose : le Visage.

L'œuvre de Levinas se présente comme la contestation incessante d'une philosophie idéaliste qui a poussé jusqu'à ses extrêmes conséquences le primat de l'ego transcendantal. Et son effort sera de poser la subjectivité comme autre chose que la pure présence à soi d'une conscience souveraine, sûre d'elle-même et irrespectueuse. Car pour lui, à définir la subjectivité par l'intentionnalité de la conscience on propose une relation du Même et de l'Autre où celui-ci ne détermine pas le Même mais est déterminé par lui. Pour aller vite on peut dire que la $5^{\mathrm{e}}$ Méditation, loin de chercher l'existence d'autrui ou sa temporalité propre fait plutôt un ultime effort pour achever 
la structure du domaine transcendent de l'ego. L'accusation de solipsisme n'est pas évacuée.

Ainsi, la subjectivité husserlienne est toujours possession ultime de soi, alors que Levinas pense la conscience comme déjà possédée par l'altérité. Non seulement par celle du monde, mais par celle d'autrui, l'autre qui me fait face dans la plus proche proximité. L'altérité husserlienne est toujours relative à ce dont ma conscience prend conscience. Pour Levinas c'est l'inverse: la conscience n'acquiert son identité qu'à partir de l'altérité. L'idée si personnelle d'allégeance à autrui impose le thème d'un moi qui ne tiendrait plus sa légitimité de soi. L'identité est une faille, une rupture, l'enjeu est d'habiter cette faille, d'assumer l'identité comme rupture incessante. Aux antipodes de la conviction idéaliste, la position du moi ne tient pas sa légitimité de soi. Elle est ébranlée par l'altérité du monde et plus encore par celle d'autrui qui fait éclater le système ou la totalité qui voudrait tout réduire au Même, au rythme synchronique de ma conscience.

En conséquence, la phénoménologie est incapable de respecter l'autre et son sens et partant, elle reste une philosophie de la violence.

À travers la rupture avec Husserl, c'est l'idéal de totalisation qui est visé, totalisation dont l'idéal porte à ne rien laisser au dehors. Interruption donc de la totalité qui témoigne de l'ouverture de la pensée à une dimension de l'esprit qui ne relève pas du savoir, interruption de la totalité qui semble revêtir une double portée: d'abord négative en ce sens qu'elle se présente comme le refus d'une métaphysique qui vise à enfermer dans un système tout élément d'extériorité, puis positive en ce sens qu'elle peut permettre une autre métaphysique définie comme le rapport à l'extériorité. C'est donc bien à une interruption de la colonisation de l'Autre et à une redéfinition de l'identité comme aptitude à se séparer de la totalité, à rompre avec une pensée qui pense à sa mesure, irruption du prochain qui demeure rupture dia-chronique, résistance du temps à la synthèse de la simultanéité, à une interruption donc d'une pensée pensant "à satiété ", toujours à son échelle, à une interruption qui permet de passer d'une pensée de... (philosophie de la violence) à une pensée pour... (philosophie de la caresse) que nous convie Levinas.

Ainsi la phénoménologie de la vie proposée par Levinas paraît sous-tendue par le projet de faire apparaître "le surgissement du moi », la subjectivité, comme une rupture de la totalité. Il s'agit donc, pour l'auteur, de montrer que cette interruption n'annonce pas la reconstitution d'un autre ordre plus complexe ou plus riche et qu'elle n'apparaît pas comme un moment nécessaire et transitoire mais bien comme une condition dont l'implication majeure est la possibilité pour l'individu de refuser le concept. L'interruption apparaitrait comme le mode d'individuation du sujet humain à proprement parler.

41 Le rapport éthique est bien la situation où la totalité se brise. Penser, au-delà du phénomène et de l'être, une épiphanie de l'Autre irréductible à toute mesure exige un bouleversement absolu des catégories par lesquelles le moi se connaît. Rencontrer l'Autre, rapport éthique, exige du moi de se laisser totalement dépayser. Dès lors la philosophie ne peut être sauvée que par l'incessant bouleversement de l'isolement absolu de chaque chose. Elle doit se résoudre au geste qui blesse la clôture du Même sur lui-même. Le projet Levinassien, dont l'analyse nous conduit vers une philosophie « de la caresse », est d'affirmer comme le dit Ricœur l'antériorité de l'éthique au train de 
l'être et de son essence, éthique qui consiste justement à accorder une priorité de l'Autre sur notre crispation à être, sur notre égoïsme ontologique.

L'enjeu éthique de cette réflexion est de parvenir à dessiner, esquisser les limites toujours fluctuantes d'un espace dans lequel autrui puisse troubler, rompre notre quant à soi toujours emprunt de raison théorique, de soif identitaire, de Même. Ce n'est qu'à ce prix que le respect de l'autre est possible. Si l'altérité se présente souvent comme une entrave à la raison théorique, l'éthique comme optique est une perspective d'intelligibilité qui ne coïncide pas forcément avec la priorité de l'évidence rationnelle sans pour autant sombrer dans l'irrationnel. Ainsi il faut monter que l'éthique peut s'approcher selon différents plans : un plan d'immanence fait justement de ruptures, ruptures avec les conceptions du monde, les systèmes de valeurs, de droits et de devoirs et un plan dans lequel peut se jouer la transcendance, le rapport à l'altérité qui, pour qu'il puisse s'accomplir et non se dominer, requiert un certain nombre d'interruptions dont certaines ont été précédemment évoquées. La relation éthique, si l'on veut, comme l'a fait Levinas notamment, lui donner un sens premier, sourd de cette interruption qu'introduit autrui, de ce malentendu primordial qui rend le dialogue possible et nécessaire, cet espace sans catégorie. «Il faut que les catégories manquent pour qu'autrui ne soit pas manqué ». Savoir s'interrompre, s'effacer pour que l'autre prenne sa place. Apprendre à interrompre le cours et le flux du vécu pour retrouver cette discontinuité du temps, seul mode de la relation à l'autre, que nous enfouissons le plus souvent sous la croûte du "continuum» d'une histoire sans histoire. Il est nécessaire d'user du recours ultime que nous ménage le paradoxe qui dénie et défie l'opinion commune, le confort des évidences, la certitude d'une foi prisonnière des scories du savoir. Rompre, ramer à contre-courant des conditions habituelles, risquer l'aventure de l'autre sans préjuger de l'échec même garanti, telle est la praxis éthique que nous propose le philosophe.

Pour Levinas le solipsisme du sujet connaissant est la structure même de la raison. Le Moi a tendance à étendre partout son identité, alors que l'éthique est l'expérience de la division qui structure le sujet; celle qui se passe entre le réel et le langage, entre le savoir et le non-savoir. C'est de l'événement de la relation que vient le sens. L'autre rompt, il est l'irruption d'une signification que ne mesure pas mon désir mais que voudrait pourtant enclore le savoir, la pensée. La relation est la fin, le principe et le médium de l'éthique. Elle se fonde et se constitue sur l'épreuve de cette interruption et de ce renversement, en vertu desquels le sujet peut retrouver les repères qu'il cherche, en renonçant à ceux qu'il a jusqu'ici pris pour des références absolues. La condition éthique est celle de tout sujet touché par l'autre qui le fait s'étonner de lui-même et en exige la responsabilité, "...responsabilité si démesurée que nous y sommes livrés passivement, à l'extrémité de toute patience, plutôt que capable d'y répondre par notre autonomie, notre prétention de Sujet, au contraire assujettis, mis à découvert... découverte risquée de soi... ${ }^{23}$. La responsabilité-pour-autrui, exigence infinie, est une contrainte et non un divertissement. "Le sujet-déposé, comme on le dit d'un tyran, se trouve exposé à l'Autre, notamment dans le langage et le Désir qu'il faut entendre comme mouvement vers autrui s'effectuant malgré soi pour l'autre: affectivité. «La subjectivité du sujet, c'est la vulnérabilité, exposition à l'affection, sensibilité, passivité plus passive que la passivité... » Autrement qu'être ou au-delà de l'essence. " ".

Il y a bien naissance du sujet, oui mais d'un sujet comme assujetti à l'autre, un sujet destitué, faible, d'une faiblesse vouée (« vouée et non pas se vouant ») à autrui. Autrui 
arrête ma liberté parce que face au visage d'autrui, je ne peux pas vivre dans l'insouciante certitude de moi-même, je suis enjoint de répondre d'autrui, d'être commandé par cette altérité contre laquelle la pensée investigatrice achoppe, contre laquelle le triomphe de la subjectivité se brise. Cette signification de l'autre n'est donc pas «con-préhensible», ne rentre pas dans les corrélations d'un système ou d'une totalité, ni même par la spiritualité occidentale telle qu'elle est mise à jour par la philosophie, c'est-à-dire le théorétique, la contemplation, le savoir. Pour lui faire droit il faudrait une pensée qui ne soit plus bâtie comme une relation, une pensée devenue incapable de s'approprier et de maîtriser son contenu. La simultanéité des termes de la relation théorique, le concept, au lieu d'être la source même de l'intelligible et de la signification, devient ici le début de la totalisation qui ruine la transcendance. Ce que Descartes appelait « idée de l'infini en nous ", fait écho, d'après Levinas, à ces exigences impossibles pour la pensée théorique (je rappelle ici que chaque fois que nous parlons de pensée théorique, de pensée conceptuelle c'est dans l'opposition à ce que nous appelons la "philosophie de la caresse ») précisément parce que l'idée de l'infini suppose un psychisme - irréductible au savoir - qui soit capable d'accueillir plus qu'il ne contient : ce qui signifie faire plus et mieux que penser : désirer. "Une pensée qui pense plus qu'elle ne pense est Désir ${ }^{25}$.

Un tel rapport à une altérité irréductible, où l'intériorité de la conscience n'a plus la structure théorétique du savoir, entre le sujet qui pense et l'idée d'infini qu'il trouve en lui s'établit une relation unique en son genre dont le modèle est le rapport avec Autrui. Ce rapport sans corrélation ou comme Levinas le dit souvent, cette "relation sans relation ", n'annule pas l'intervalle de la séparation entre ses termes et par conséquent ne détruit pas la transcendance. Seulement, pour le rappeler encore, la transcendance n'est plus ici une couche d'être ou une dimension de réalité au-delà de l'expérience subjective, elle se niche au sein même de la subjectivité dans la concrétude même du psychisme préalable au savoir. Avant le savoir, le Même (ma sphère identitaire) est déjà mis en question par l'Autre qui se révèle sans se dévoiler, sans se réduire à un moment provisoire de la synthèse théorique dont la pensée peut difficilement se passer. Le face à face de la proximité est une relation irréductible à la totalisation du savoir.

Sans confondre la transcendance avec l'extase mystique qui dépersonnalise le sujet et l'abîme dans l'Autre il faut chercher, tout en suivant scrupuleusement la méthode de la phénoménologie descriptive, une relation qu'il étaye comme sujet - mais sans être sujet de savoir et de pouvoir - qu'il étaye donc, dans sa passivité, indépendamment des rapports s'établissant dans la collectivité sociale et politique. Cette relation c'est principalement la relation éthique, en particulier la relation amoureuse, érotique. Audelà du savoir et du pouvoir, dans l'éros aussi bien que dans l'ethos une ipséité irremplaçable est impliquée dans une relation à autrui qui, au lieu d'abolir la séparation des termes et de se faire simultanéité synchronique, se nourrit de cette dualité dia-chronique non synthétisable et qui constitue en propre la dimension de respect.

Ce qui importe dans le rapport à l'autre, également pour l'autre de l'amour et du désir, c'est son imprévisibilité, son inconnaissabilité même, le fait que l'autre, même dans un amour éternel, nous demeure toujours autre. Dans l'amour, l'autre est accueilli comme une altérité infinie, une altérité dont on ne peut faire ni le tour, ni le calcul. Être en relation avec l'autre et en particulier si cette relation est placée sous le signe de l'amour, c'est accepter de n'en avoir jamais fini avec lui. Et c'est bien parce que 
l'intentionnalité de l'amour, si vraiment nous pouvons parler d'intentionnalité, est de s'exposer à cette infinitude que l'amour et la relation à autrui ne demeure vivants qu'à force de récuser toutes les images comme les formes de la connaissance conceptuelle dans leurs dimensions communes de cadrage, d'immobilité, de catégorisation. « Il est frappant que l'être que nous aimons soit celui dont nous avons le plus de peine à dire comment il est. Nous l'aimons simplement. [...] l'amour nous libère de toutes images. Ce qui fait de lui une aventure passionnante et risquée, c'est que nous n'en avons jamais fini avec les êtres que nous aimons, justement parce que nous les aimons et tant que nous les aimons ${ }^{26}$. amoureux. Celui-ci scrute, épie, inspecte et tout le visage aimé sollicite sa vigilance [...]. Le visage aimé est un fouillis de signes entre lesquels il a perdu le pouvoir de faire le tri [...]. L'art n'est pas l'exutoire de l'amour qui est la religion du visage qui en interdit la représentation [...], il n'est ni beau, ni sublime, il est différent d'une splendeur ineffable, d'un chef-d'œuvre qui ne se laisse pas décrire pour être une présence qui ne se laisse pas enclore ${ }^{27}$. Le moment où nous pensons connaître l'autre est toujours, déjà, la fin de l'amour. Seulement il n'en va pas ici comme on croit d'habitude : ce n'est pas, en effet, parce que nous connaissons l'autre que notre amour s'éteint, mais à l'inverse, parce que notre amour s'éteint, parce que sa force s'épuise, que sa résistance s'amenuise, l'autre cesse alors d'avoir du prix pour nous et sa valeur éthique d'interpellation, d'assignation à répondre, à présence, s'étiole. Dès lors nous lui refusons ce que demande tout être vivant, c'est-à-dire demeurer insaisissable et en même temps nous nous étonnons et bien entendu nous sommes déjà déçus que notre relation ait cessé d'être vivante. Nous sommes fatigués d'affronter le mystère que l'autre ne cesse pas d'être, nous nous faisons une image : voilà le geste sans amour, la trahison, l'irrespect.

Toutefois cette trahison n'est pas innocente, il arrive bien souvent qu'elle procède d'un acte volontaire. Il y a en effet la volonté de l'amant de voir l'autre dans la fixité d'une idole, la volonté de voir le mouvement de son visage se stabiliser en beauté, c'est-à-dire idolâtrie esthétique, la volonté de stopper la dérobade infinie de l'autre. L'amour, si passionné soit-il, est fatigant. Le sommeil par exemple statufie le visage qui, dès lors, n'est plus ni voix ni regard et par-là même consent à l'immobilité et devient prévisible, c'est-à-dire devient masque. L'amoureux alors peut troquer le tourment pour la contemplation et ainsi se reposer de l'amour. Or ceci est un vœu pieux dans la mesure même où il n'y a d'amour que dans l'impossibilité d'arrêter la dérobade infinie de L'autre, de fixer son imprévisibilité, d'enliser sa transcendance. Cet enlisement, cette fixité ne procèdent proportionnellement que de la décadence de l'amour. Le regard se ré-esthétise à mesure que s'épuise la passion, si bien qu'à la fin, lorsque l'amour n'est même plus une trace même un simple souvenir, l'autre se fige, s'immobilise dans son effigie, chute au rang d'image. Quand il est gagné par l'épuisement, le visage aimé devient figure et me laisse là " comme un déchet » sans que je puisse rien faire contre cet abandon.

Aimer l'autre plutôt que de le penser ou de le connaître c'est, à l'encontre de ce que disait Bertold Brecht, (« aimer quelqu'un c'est se faire une esquisse (Entwurf) »), faire un projet, c'est vouloir transformer le monde entier, non plus cette fois-ci sous l'horizon de la pensée qui thématise et conceptualise, mais en faisant des choix tout en sachant que l'on court le risque de l'imprévisibilité même de l'autre. Alors même que nous 
sommes poussés par l'irrésistible désir de vouloir, l'instant d'une analyse, que l'autre retourne à la lumière et soit assigné à cette loi commune qu'est la définition, dans l'amour, l'amoureux lâche la proie pour l'ombre, le savoir pour le désir. Dans le face à face amoureux, l'être aimé demeure transcendant par rapport à moi. "Le "ne pas connaître" n'est pas ici à comprendre comme une privation de connaissance [...], ce n'est pas comme un raté du savoir que l'amour est amour $"{ }^{28}$. Dans l'amour, l'autre arrive du dehors, s'installe en nous et nous demeure étranger. Il nous atteint jusqu'à occuper, accaparer tout le champ de la conscience et pourtant continue à se dérober à notre atteinte, il entre en nous dans une intrigue, un mystère qui tranche sur le savoir. C'est pourquoi, dans le dessaisissement qui caractérise l'état amoureux, subsiste toujours quelque part la volonté de compenser cette dérobade par le savoir. Dans l'amour ; rien ne nous protège plus du visage d'Autrui. Aimer, suprême passivité, c'est s'exposer et se soumettre. L'amour fait de nous l'otage d'un absent que l'on ne peut fixer, ni esquiver, ni éconduire, il vise l'énigme même de l'autre qui n'est jamais réellement ni mon égal ni mon contemporain.

51 Mais la condition à tout ceci, c'est que cette dimension n'est intérieure à l'amour qu'aussi longtemps où elle ne se fige pas en image et dans la mesure où on est capable de projeter, justement par delà les images, un horizon non neutre d'altérité sous lequel seulement l'autre peut être aimé "pour lui-même » au-delà de toutes ses qualités comme de toutes nos attentes. Ainsi nous pouvons dire que la proximité conjugale n'abolit en aucune manière la distance, elle en supprime seulement les causes accidentelles. Ce que l'on trouve avant tout dans la proximité, c'est l'inquiétude, la tendresse et le désir qui consistent, comme le dit Levinas, à " poursuivre ce qui est déjà présent à chercher encore ce que l'on a déjà trouvé " ${ }^{29}$, "à solliciter ce qui échappe sans cesse de sa forme ${ }^{30}$. Ainsi dans l'amour, la présence est une modalité de l'absence et c'est cela qui constitue à la fois le désespoir de l'amoureux mais également son trésor le plus cher, sa richesse la plus grande.

Contre Sartre, Levinas, lui, découvre dans la dualité insurmontable de la relation érotique non pas les manœuvres d'un conflit violent et irrespectueux, mais le pathétique de l'amour, le voluptueux même de la volupté, le vulnérable. Dans le modèle de Sartre, en effet, la caresse, symbole de la relation érotique, est avant tout envisagée comme un façonnement et la vie elle-même comme un roman où tout est combat, où derrière les moments les plus tendres, derrières les mélodies câlines de la fusion des corps, transparaît la lutte même des consciences. Avec une rigueur sans merci la description phénoménologique sartrienne met à jour l'agressivité et les machinations qui s'ourdissent derrière l'immanence de la caresse. «La caresse n'est pas un simple effleurement : elle est façonnement. En caressant autrui, je fais naître sa chair, par ma caresse, sous mes doigts. La caresse est l'ensemble des cérémonies qui incarnent autrui » ". Dès lors, la caresse apparaît ou plutôt est envisagée comme une embuscade tendue à l'autre pour qu'il se fasse présence offerte, renonçant par-là à son regard et à sa liberté. La caresse se présente donc comme une invitation à la passivité la plus radicale, elle est la tentative désespérée du désirant pour empâter l'être désiré dans sa chair, pour qu'il ne s'échappe plus et l'ultime effort pour se dessaisir de son regard, pour cesser de vivre sous son regard. Ainsi, aussi tendre ou fervente soit-elle, la caresse est animée du désir de rendre l'autre inoffensif, de le désarmer, de le transformer en objet afin de pouvoir l'enclore et l'enfermer au lieu qu'il me transcende de toutes parts dans les limites de son pur être là. «Chez Sartre la caresse est revanche, désir de 
devenir propriétaire. Par la caresse je fige et j'entraîne à mon tour dans l'inertie celui ou celle dont le regard m'a englué dans l'être $»^{32}$.

Il est possible, en outre, de croire dans un premier temps que la position de Sartre concorde à certains égards avec les déterminations de la pensée de Levinas dans le sens où, dans les deux cas, l'amour apparaît comme ce qui est, en propre, incompatible avec toute possession et avec toute connaissance : «[...] celui qui veut être aimé ne désire pas l'asservissement de l'être aimé, [...] il arrive qu'un asservissement de l'être aimé tue l'amour de l'amant. Le but est dépassé: l'amant se retrouve seul si l'aimé s'est transformé en automate. Ainsi l'amant ne désire-t-il pas posséder l'aimée comme on possède une chose ", mais la suite du texte marque une rupture radicale avec celui de Levinas:» il réclame un type spécial d'appropriation, il veut posséder une liberté comme liberté " ${ }^{33}$. Ainsi chez Sartre la caresse chercherait à dominer une «liberté hostile » et à lui arracher son consentement. De cette façon l'analyse sartrienne que vise le texte de Levinas fait du "conflit » le sens originel de «l'être-pour-autrui » et voit dans ce conflit un projet d'unification qui poursuivrait la disparition du caractère d'altérité d'autrui, son identification à moi, c'est pourquoi le rapport à autrui consisterait avant tout à «agir sur la liberté d'autrui » pour la nier comme telle, pour « opérer l'assimilation à moi de l'autre liberté ».

Dans un deuxième temps, Levinas, contre le modèle fusionnel cette fois-ci de la relation érotique, exalte la séparation des êtres dans la rencontre des corps. L'Éros n'est plus le théâtre éphémère où s'efface la discontinuité entre les individus, mais bien le moment où s'ouvre un abîme vertigineux. Il n'y a pas, avec Levinas, de communion érotique, de fusion. Ce que découvre au contraire le désir c'est l'indomptable et immaîtrisable "proximité » de l'Autre. Dans le rapport à l'aimé ou à l'aimée il n'y a aucune échappatoire : rien en lui ne me distrait de son altérité, le corps sous la caresse « se fait tout entier visage ». Ainsi l'autre n'est pas un objet que je m'approprie ou une liberté que je dois circonvenir pour affirmer avec violence la mienne : c'est un être dont le mode d'être consiste à ne jamais complètement se livrer, que se soit à la convoitise, à la connaissance ou encore au regard. Avant d'être violence ou profanation, l'érotisme est l'expérience de l'inviolabilité éthique d'autrui ou mieux encore, sa pudeur. Nous l'avons signalé, Levinas n'accepte pas de poser l'amour comme une "fusion ». "Le pathétique de l'amour consiste en une dualité insurmontable des êtres. C'est une relation avec ce qui se dérobe à jamais. La relation ne neutralise pas ipso facto l'altérité mais la conserve. Le pathétique de la volupté est dans le fait d'être deux, mais l'autre n'est pas ici un objet qui devient nôtre ou qui devient nous, il se retire au contraire dans son mystère $»^{34}$.

La relation érotique n'est donc ni une lutte, ni une fusion, ni une connaissance. Il faut reconnaître avec Levinas sa place exceptionnelle parmi les relations car la relation érotique se présente bien comme la relation avec l'altérité, avec le mystère, c'est-à-dire avec l'avenir, avec ce qui, dans un monde où tout est là, n'est jamais là, avec ce qui peut ne pas être là quand tout est là. Non pas avec un être qui n'est pas là, mais avec la dimension même de l'altérité. Nous pouvons en effet considérer de rapport avec l'autre par l'éros comme un échec et on aura raison si on adopte la terminologie des descriptions courantes et si l'on veut caractériser l'érotique par le "saisir», le " posséder », le « connaître ». Or il n'y a rien de tout cela ou échec de tout cela dans l'éros. Si on pouvait, en fait, posséder, connaître et saisir l'autre il ne serait plus l'autre. D’ailleurs, le plus souvent, le rapport avec l'autre est généralement, dans la perspective 
qui nous concerne, recherché comme une fusion. Mais la relation à l'autre, en définitive, c'est l'absence de l'autre, mais pas absence pure et simple, non pas absence de pur néant, mais absence dans un horizon d'avenir, une absence qui est celle du temps lui-même, un horizon que seule réintroduira la dimension érotique.

L'éros, dans son étrangeté même, a la faculté de bouleverser, il bouleverse, il est bouleversement de la subjectivité définie comme relation avec les êtres que nous identifions, bouleversement de la conscience de soi où nous nous identifions à nousmême. Il est, on peut le dire, destitution de la conscience face à sa propre fonction constituante.

Ainsi, dans l'érotique, la caresse opère une déconstruction et cette déconstruction ouvre sur «la profondeur vertigineuse de ce qui n'est pas encore, qui n'est pas, mais d'une existence n'ayant même pas avec l'être la parenté qu'entretient avec lui une idée ou un projet». [...] «La caresse ne vise ni une personne, ni une chose, on peut dire qu'elle vise le rien qui n'a plus le statut d'un "étant », qui, sorti des « nombres » et des "êtres » n'est même pas la qualité d'un étant ». La caresse se présente donc comme le degré zéro de signification et ce degré zéro serait comme la virginité elle-même, la fragilité même de ce qui se trouve à la limite du non-être, limite où ne se loge pas seulement ce qui n'est plus, mais également et surtout, ce qui n'est pas encore. Expérience à la limite, voilà ce qu'est la caresse, volupté, désir. Volupté qui ne comble pas le désir mais qui est le désir lui-même. Degré zéro de l'être, la volupté est donc une expérience pure une expérience qui, comme nous allons le voir, ne se coule dans aucun concept, une expérience qui demeure, envers et contre tout, aveuglement, expérience pure.

Dès lors, la caresse se présente comme un éclatement, une transcendance vers l'autre, qui, lorsqu'elle s'en approche, ne se renferme pas comme une prise, ne se renferme pas en une emprise, en un " main-tenant ». "La caresse est un mode d'être du sujet où le sujet, dans le contact d'un autre, va au-delà de ce contact. Le contact en tant que sensation fait partie du monde de la lumière. Mais ce qui est caressé n'est pas touché à proprement parlé ${ }^{35}$. La caresse découvre donc avant toute chose une intention, une modalité d'être qui ne se pense pas dans son rapport au monde comme saisir, posséder ou connaître. Elle n'est pas et ne se présente pas comme un savoir mais comme une expérience, une rencontre. Sans être ni pouvoir, ni violence, ni fusion elle est en définitive: tendresse, relation, respect et vulnérabilité. Se présentant comme philosophie, (philosophie de la caresse comme philosophie de la déconstruction), la caresse déconstruit le sens et produit une non-signifiance qui se tient dans un «no man's land» entre l'être et le "ne-pas-encore-être». La philosophie de la caresse ébranle les perceptions univoques et finies où la pensée est déjà toute faite, où tout est instauré une fois pour toute. Critique de la raison dogmatique, la caresse est un relativisme, un scepticisme sans nihilisme.

Elle dénonce, en fait, les défauts du concept qui résultent d'une fixation et d'un durcissement de la pensée. En effet, la réduction du concept vise un «être-ensemble » qui repose sur une entente. Le concept est né pour éviter les "malentendus», le concept manque, dans la généralisation, les différences individuelles des choses et des hommes. Il ne respecte rien. Dans le monde du concept, monde de la prise (la griffe du Begriff), la force fondamentale est une dynamique immanente de la mise en demeure où la communication est réduite à la mise en relation de « moi » substantiels et, semble-til, exclut le dialogue où pourrait s'exprimer transcendance et altérité. Le monde du 
concept, prise et emprise, est le monde du «main-tenant », annulation de la possibilité même du temps. Il est toujours présent comme quelque chose de représentable, de communicable, d'explicable, d'accumulable. Le monde du concept apparaît toujours pour Levinas comme le résultat de l'impuissance originaire à supporter un monde qui serait pur chaos, devenir contradictoire, informe et informulable. Contre l'être en devenir la temporalité de la prise du concept est celle de la présence, de l'instant sorti du flux du temps, instant figé, gelé à la limite du hors temps : présent éternel.

La philosophie de la caresse, nous l'aurons compris, toute empreinte de respect et de vulnérabilité, a pour objectif de faire obstacle au dogmatisme de la raison et de mettre en place une sorte de " philosophie du non ", représentant par là, nous semble-t-il, une attitude critique par rapport aux interprétations du monde. Elle permet le recul et la prise de conscience du caractère relatif de toutes les interprétations explicatives et de toutes les significations. La relativité de la caresse exclue ce que Levinas appelle la vérité totalitaire et incarne, pour reprendre son expression: "la sagesse de l'incertitude ». Parce que la caresse est cette rencontre avec l'absolument autre, elle n'est ni représentation, ni relation conceptuelle au même. Ni le moi, ni l'autre de la rencontre ne se laissent totaliser par le concept réducteur de relation. La rencontre qu'occasionne la caresse, est la seule aventure hors de soi, vers l'imprévisible. Résistance à la conceptualité, désordre de celle-ci, la rencontre est possible par l'autre, par son imprévisibilité " réfractaire à la catégorie ». Le concept, en effet, suppose une anticipation, un horizon où l'altérité s'amortit en s'annonçant et, par là même, suppose de se laisser prévoir, de ne plus être respectée. L'infiniment autre ne se laisse pas relier dans un concept, ne se laisse pas penser dans un horizon qui est toujours, selon Levinas, horizon du même où plus rien de surprenant n'advient. "L'extraordinaire commence au moment où je m'arrête " pourrait-on dire avec Blanchot dans L'Arrêt de mort. "Sans doute cette rencontre, qui pour la première fois n'a plus la forme du contact intuitif, mais celle de la séparation, sans doute cette rencontre de l'imprévisible lui-même estelle la seule ouverture possible du temps, le seul avenir pur ${ }^{36}$.

61 La caresse, donc, nous l'avons précisé, déconstruit le sens et produit une nonsignifiance qui se tient, à l'écart du concept, dans un «no man's land» entre l'être et le « ne-pas-encore-être ». Désignification du sens, du moi, l'herméneutique de la caresse conduit dans un «chemin qui mène nulle part». La caresse «ne saisit rien, n'aboutit pas, n'a ni la structure sujet-objet, ni la structure moi-toi. L'Éros ne s'accomplit pas comme un sujet qui fixe un objet ni comme une projection vers un impossible. Son mouvement consiste à aller au-delà du possible ${ }^{37}$.

Il nous paraît important de préciser que la caresse procède non pas du besoin mais bien du désir. "Dans le besoin, argumente Levinas, je puis mordre sur le réel et me satisfaire, assimiler l'autre. Dans le désir pas de morsure sur l'être, pas de satiété, mais avenir sans jalons devant moi $"{ }^{38}$. Tandis que le besoin, dont le corrélat pour Levinas est la jouissance, ne brise pas l'immanence et n'est qu'un "mouvement du Même », le "remous du Même ", le désir, lui, est la volupté même. Pour Derrida, ce concept de désir apparaît comme aussi anti-hégélien que possible: il n'est en aucun cas mouvement de négation et d'assimilation nécessaire pour devenir « conscience de soi » par exemple. Le désir est au contraire le respect de l'autre comme tout autre, moment éthico-métaphysique que la conscience doit s'interdire de transgresser. Le désir, le mouvement du désir ne peut être ce qu'il est que comme renoncement au désiré. Contrairement au besoin qui émane ou procède de la sphère immanente, le désir se 
laisse appeler par l'extériorité absolument irréductible de l'autre auquel il doit rester infiniment inadéquat, par rapport auquel il doit demeurer toujours en retard. C'est pourquoi la métaphysique du désir est donc une métaphysique de la séparation infinie.

Ainsi la caresse ne se saisit de rien, "elle consiste à ne se saisir de rien». Son intentionnalité, c'est-à-dire l'intentionnalité érotique, ne culmine pas dans l'éclosion d'une quelconque présence, telle, par exemple, cette pure présence de l'éternité intelligible qui caractérise l'Idée de Beauté chez Platon. Ainsi, curieusement, dans la relation érotique, chacun chemine vers quelque infini qui, plutôt que de se laisser enclore dans la pensée et le discours conceptuel, palpite au contraire dans la rencontre sans se clore ni se décider selon l'échéance de la dimension d'un quelconque transcendant à rejoindre. L'Aimé, sous la caresse, entraîne l'amant dans un abîme vertigineux pour que, de ces profondeurs, il se laisse emporter dans un avenir absolu. La caresse ne s'accomplit pas dans la saisie d'une plénitude de présence, car elle s'attache à quelque chose qui « s'échappe sans cesse vers un avenir ». Elle est " visée de ce qui se dérobe comme s'il n'était pas encore ». La caresse se présente donc comme une transcendance sans télos: "elle cherche, elle fouille», elle est avant toute chose une pure quête, un pur mouvement de transcendance sans issue possible dans la contemplation d'un transcendant quel qu'il soit. Respect du vulnérable car vulnérabilité respectueuse.

\section{NOTES}

1.. « Notre compagnie clandestine ", in Textes pour E. Levinas, 1980, p. 81.

2. Voir à ce sujet l'étude de H. CHOPLIN sur Levinas et Laruelle, De la Phénoménologie à la non-philosophe, éd. Kimé, « Bibliothèque de non-philosophie », 1997.

3.. M. BLANCHOT, op. cit., p. 85.

4.. Ibid.

5.. LEVINAS, Humanisme de l'autre homme, Paris, Fata Morgana, 1973.

6.. M. BLANCHOT, op. cit.

7.. De Dieu qui vient à l'idée, Vrin, p. 105.

8.. En découvrant l'existence Husserl et Heidegger, Vrin, p. 175.

9.. Totalité et Infini, Essai sur l'extériorité, La Haye, Nijhoff, p. 43.

10.. Ibid., préface.

11.. Autrement qu'être ou au-delà de l'essence, La Haye, Nijhoff, p. 81.

12.. Ibid., p. 71.

13.. En découvrant l'existence avec Husserl et Heidegger, op. cit., p. 259.

14.. Autrement qu'être ou au-delà de l'essence, op. cit., p. 110.

15.. Ibid., p. 112.

16.. À la question : «Mais l'autre n'est-il pas responsable de moi ? "Levinas répond : «Je suis responsable d'autrui sans attendre de réciproque, dût-il m'en coûter la vie. La réciproque, c'est son affaire. C'est précisément dans la mesure où entre autrui et moi la relation n'est pas réciproque, que je suis sujetion à autrui ; et je suis « sujet » 
essentiellement en ce sens [...]. Le moi a toujours une responsabilité de plus que tous les autres ", Éthique et infini.

17.. Méditations cartésiennes, $§ 41$, Vrin, p. 141.

18.. LEVINAS, Entre nous, p. 84.

19.. Totalité et infini, op. cit., p. 61.

20.. Transcendance et intelligibilité, p. 13.

21.. « De la conscience à la veille », De Dieu qui vient à l'idée, op. cit., p. 42.

22. Totalité et infini, op. cit., p. 65.

23.. M. BLANCHOT, op. cit., p. 83.

24.. François PoIRIÉ, E. Levinas, La Manufacture, p. 21.

25.. En découvrant l'existence avec Husserl et Heidegger, op. cit., p. 174.

26.. MaX FRISCH, Gesammelte Werke, Frankfort, 1976, T. 2, p. 369.

27.. A. FINKIELKRAUT, La Sagesse de l'amour, Paris, Gallimard, 1984, p. 56 sq.

28.. LEVINAS, Éthique et infini, Paris, Fayard, 1982, p. 69.

29.. LEVINAS, En découvrant l'existence avec Husserl et Heidegger, op. cit., p. 230.

30.. LEVINAS, Totalité et infini, op. cit., p. 235.

31.. SARTRE, $L$ 'Etre et le Néant, Tel, p. 440.

32.. A. FINKIELKRAUT, op. cit., p. 27.

33.. SARTRE, op. cit., p. 416.

34.. LEVINAS, Le Temps et l'Autre, PUF, 1982.

35.. LEVINAS, Le Temps et l'Autre, op. cit., p. 82.

36.. DERRIDA, « Violence et métaphysique, Essai sur la pensée de Levinas ", in Écriture de la différence, Paris, Seuil, p. 142.

37.. LEVINAS, Totalité et infini, op. cit., p. 238.

38.. Ibid., p. 89.

\section{RÉSUMÉS}

Contre la prétention hégémonique de la conscience telle qu'elle se définit traditionnellement dans la philosophie, en particulier dans la phénoménologie husserlienne, contre la prétention de la subjectivité à s'universaliser, Levinas entend renverser la violence d'une pensée qui pense pouvoir tout habiter en une sagesse de l'incertitude qui trouve son origine dans une subjectivité entendue comme vulnérabilité, condition de toutes formes de respect, comme non-indifférence à la présence traumatisante de l'autre dans sa radicale altérité.

Against the hegemonic pretension of consciousness, as it is traditionally defined in philosophy and particularly in Husserl's Phenomenology, against the pretension of subjectivity to selfuniversalising, Levinas intend to change the violence of a self-satisfied thought into a wisdom of uncertainty which originates from subjectivity, considered as vulnerability, the condition to any form of respect, as non-indifference toward the trauma linked to the other in its radical alterity. 


\section{AUTEUR}

\section{NICOLAS ANTENAT}

Nicolas Antenat, professeur de philosophie, travaille, dans une approche phénoménologique, sur les rapports entre éthique et esthétique et s'intéresse à la problématique de l'interruption. 